\title{
Addendum to 'Approximations for Solute Transport Through Porous Media with Flow Transverse to Layering'
}

D. A. BARRY

Soil and Environmental Sciences Dept., University of California, Riverside, CA 92521-0424, U.S.A.

J. - Y. PARLANGE

Agricultural Engineering Dept., Cornell University, Ithaca, NY 14853-5701, U.S.A.

R. RAND

Theoretical and Applied Mechanics Dept., Cornell University, Ithaca, NY 14853, U.S.A.

and

J. C. PARKER

Soil and Environmental Sciences, Agronomy Dept., Virginia Polytechnic Institute and State University, Blacksburg, VA 24061, U.S.A.

(Received: 26 May 1987)

Key words. Convection-dispersion model, time moments.

Recently, Barry and Parker (1987) examined the convection-dispersion model for a system consisting of well-defined, homogeneous layers. In that paper the effect of switching layers in a two-layer system on time moments of breakthrough curves was considered. The analysis was based on comparison of time moments computed for breakthrough curves from a system with a semi-infinite second layer. It was concluded that the second time moments were dependent, albeit in many cases weakly, on layer ordering. The moments used in this comparison were calculated with the assumption that the remainder of the second layer, extending beyond the sampling point, had no upstream effect. If this were true, however, the physical properties of the medium beyond the sampling position could be chosen arbitrarily. Consequently, the same result should be obtained if the medium beyond the sampling point has negligible dispersive properties. This point is important since it often happens in practice that the parameters for the convection-dispersion equation are obtained from the analysis of laboratory column breakthrough curve data, in which case consideration of the physical system is necessary. For example, for an in-line filter in a continuous fluid stream, negligible dispersion in the outgoing fluid stream is a more suitable assumption. The system may then be envisioned as a three-layer medium, with the third layer 
representing an exit line with negligible dispersion. We repeat the calculations for this situation.

Consider then a layered profile consisting of three layers, with the first layer extending from 0 to $L$, the second from $L$ to $x$, and the third from $x$ to $\propto$, where the notation follows that used previously. Each layer has a retarded dispersion coefficient, $D_{i}$, as employed in Barry and Parker (1987), and retarded mean pore water velocity, $v_{i}$, for $i=1,2,3$. We again wish to examine the effect of layer ordering, i.e., we will switch layers 1 and 2 and observe changes, if any, in the calculated time moments. Since we are concerned with breakthrough curves as described above, we take $D_{3}=0$. If a Dirac source is applied at the origin at $t=0$ then the mean, variance and skewness are obtained as in Barry and Parker (1987), with one more layer. The mean, $\bar{\mu}$, at the measuring location $x$ is, as expected,

$$
\bar{\mu}=l_{1}+l_{2}
$$

where $l_{1}=L / v_{1}$ and $l_{2}=(x-L) / v_{2}$. The variance, $\mu_{2}$, is,

$$
\mu_{2}=2\left[d_{1} l_{1}+d_{2} l_{2}-d_{1}^{2}\left(1-p_{1}\right)-d_{2}^{2}\left(1-p_{2}\right)+d_{1} d_{2}\left(1-p_{1}\right)\left(1-p_{2}\right)\right]
$$

where $d_{i}=D_{i} / v_{i}^{2}, i=1,2, p_{1}=\exp \left(-v_{1} L / D_{2}\right)$ and $p_{2}=\exp \left[-v_{2}(x-L) / D_{2}\right]$.

A comparison of (2) with the corresponding expression in Table I of Barry and Parker (1987) shows that the difference between the expressions is $-2\left[d_{2}^{2}\left(1-p_{2}\right)+\right.$ $\left.d_{1} d_{2} p_{2}\right]$, that is, the value given by (2) is less than that given previously. This difference is in the anticipated direction since here it has been assumed that there is a closed boundary at the measuring location, removing the possibility of upstream dispersion of solute that has moved beyond that point. Thus, given that a homogeneous third layer exists, this comparison demonstrates that its dispersive properties will affect time moments calculated at $x$. In this context the expressions given in Table I of Barry and Parker (1987) are for the case where the second and third layers have identical dispersive properties. Depending on the parameter values, sizable errors are possible in, e.g., parameter estimation, if an inappropriate expression is used in data analysis.

The mean and variance given by (1) and (2) are for the layer ordering 1 followed by 2 . If we now switch these layers it can be seen that the mean and variance are unaffected. This is in contrast to the previous result where the variance was affected slightly by the ordering of the layers. It is now clear that to quantify the effect of switching layer ordering it is necessary to consider explicitly the 'downstream' dispersion coefficients. The previous result given by Barry and Parker (1987) was obtained ignoring this effect and is therefore not valid in general.

The symmetry displayed by (1) and (2) for the mean and variance extends to the skewness, $\mu_{3}$, as well. We have, at $x$, 


$$
\begin{aligned}
\mu_{3}= & 12\left\{2\left[d_{1}^{2} l_{1}+d_{2}^{2} l_{2}-d_{1}^{3}\left(1-p_{1}\right)-d_{2}^{3}\left(1-p_{2}\right)\right]+\right. \\
& +d_{1} d_{2}\left(1-p_{1}\right)\left(1-p_{2}\right)\left(l_{1}+l_{2}+d_{1}+d_{2}\right)-\left(d_{1} l_{1}+d_{2} l_{2}\right) \times \\
& \times\left[\left(1-p_{1}\right) d_{1}+\left(1-p_{2}\right) d_{2}\right] .
\end{aligned}
$$

In conclusion, we found that the result given by Barry and Parker (1987) concerning the effect of layer ordering on time moments was based on an incorrect assumption. A reanalysis of the convection-dispersion model with constant parameters shows that the mean, variance, and skewness calculated for the time moments of the breakthrough curve from two finite layers are unaffected by the layer ordering for the important practical case considered here.

\section{Reference}

Barry, D. A. and Parker, J. C., 1987, Approximations for solute transport through porous media with flow transverse to layering, Transport in Porous Media 2, 65-82. 